selectively occurred near sites of inflammation. Importantly, neither TREM2-transduced nor control BM-MCs migrated into the CNS of healthy mice or EAE mice in the early stages of clinical disease; migration was only observed when EAE symptoms were at their peak.

The results of these experiments indicate that TREM2-transduced myeloid precursor cells facilitate recovery and repair by resolving inflammation and encouraging debris clearance. Further animal studies are needed before this approach can be considered for clinical applications, but it potentially opens up new targets for MS therapy after the onset of symptoms.

Original article Takahashi K et al. (2007) TREM2transduced myeloid precursors mediate nervous tissue debris clearance and facilitate recovery in an animal model of multiple sclerosis. PLoS Med 4: 675-689

\section{Increased antithrombotic use puts the over 75s at risk for stroke}

UK stroke mortality data indicate a decline in hemorrhagic stroke incidence over the past 20 years in individuals aged $<75$ years. Trends for intracerebral hemorrhage $(\mathrm{IH})$ in older individuals are unknown, but are likely to differ from those in younger people, as the predominant etiology of stroke alters with age. Lovelock et al. have compared data from the Oxford Community Stroke Project (OCSP; conducted 1981-1986) with data from the Oxford Vascular Study (OXVASC; 2002-2006) to examine changes in the incidence of $\mathrm{IH}$ over time.

The overall standardized incidence of all $\mathrm{IH}$ decreased over time. This decrease was, however, mostly attributable to a reduction in $\mathrm{IH}$ incidence in individuals aged $<75$ years; little change was noted in the incidence of $\mathrm{IH}$ in individuals aged $\geq 75$ years. The incidence of hypertensionassociated $\mathrm{IH}$ fell in both age-groups, but among those individuals aged $\geq 75$ years, there was a considerable rise in the proportion of patients without hypertension who had an antithrombotic bleed. This finding corresponds with the marked difference in antithrombotic use between OCSP and OXVASC (4\% vs $41 \%)$.

The authors conclude that, while efforts to treat hypertension have reduced the incidence of $\mathrm{IH}$ in individuals under 75 years of age, they have had little effect in older individuals, in whom antithrombotic use seems to be a more important risk factor. Given the aging population in the UK, the absolute number of cases of $\mathrm{IH}$ might increase if the risk associated with antithrombotic use is not addressed.

Original article Lovelock CE et al. (2007) Change in incidence and aetiology of intracerebral haemorrhage in Oxfordshire, UK, between 1981 and 2006: a populationbased study. Lancet Neurol 6: 487-493

\section{Gabapentin reduces pain severity in patients with fibromyalgia}

Gabapentin, a structural analog of the neurotransmitter GABA, has antinociceptive properties in patients with hypersensitized pain conditions. Arnold and colleagues, therefore, assessed the safety and efficacy of gabapentin in patients with fibromyalgia.

This randomized, double-blind, placebocontrolled trial included patients who met the American College of Rheumatology criteria for fibromyalgia and who scored $\geq 4$ on the Brief Pain Inventory (BPI) average pain severity score. Patients (90\% women) were randomly allocated to receive either gabapentin $(n=75)$ or placebo $(n=75)$. Gabapentin dose started at $300 \mathrm{mg}$ per day for week 1 , and was increased incrementally up to $2,400 \mathrm{mg}$ per day at week 6 . This dose was tapered by $300 \mathrm{mg}$ per day if it was poorly tolerated (to a minimum of $1,200 \mathrm{mg}$ per day); dosage was kept stable for the final 4 weeks of the 12 week therapy phase. Patients were assessed every 1-2 weeks and at the end of treatment.

Significantly more patients in the gabapentin group achieved a response at the end of the study (defined as $\geq 30 \%$ reduction in $\mathrm{BPI}$ average pain severity score) than in the placebo group (51\% versus 31\%, $P=0.014)$. In addition, significantly more gabapentin-treated patients experienced improvements in sleep disturbance than placebo-treated patients. Adverse effects were mostly mild or moderate; the frequency of serious adverse events was not significantly different between groups.

The authors conclude that gabapentin is an effective short-term treatment for the pain symptoms of fibromyalgia; further clinical trials are required to evaluate its long-term efficacy for this disease.

Original article Arnold Let al. (2007) Gabapentin in the treatment of fibromyalgia: a randomized, double-blind, placebo-controlled, multicenter trial. Arthritis Rheum 56: 1336-1344 